Vol. 5, No. 3-4, 2019

Vitalii Molchanov

Department of Technology of Engineer, Dniprovsky State Technical University, Ukraine, Dnipropetrovsk region, Kamianske, Dniprobudivska Street, 2, E-mail: v_molchanov@ukr.net

\title{
STATEMENT AND SOLUTION OF NON-STATIONARY PROBLEM OF LIQUID FILTERING IN A DEFORMED POROUS MEDIUM
}

Received: December 2, 2019 / Revised: December 27, 2019 / Accepted: December 28, 2019

(C) Molchanov V., 2019

Abstract. The paper discusses the laws behind the filtering procedures of process liquids through porous materials. At metalwork finishing operations, the use of cutting fluids is of particular importance. During operation, liquids are continuously and intensively contaminated with solid metal parts. To restore the original properties, process fluids are cleaned of mechanical admixtures. The most widely used methods for purifying process liquids are those by filtration. The use of filtration for the purification of process fluids is most effective, since filtering through a layer of porous materials results in complete extraction and removal of solids from liquids. However, the structural features of the pores in the porous environment trigger a number of specific phenomena that arise when liquids move in the porous channels of a porous medium.

The research purpose is to discuss and establish the laws behind the filtering procedures of process fluids through porous materials. When filtering process liquids through a layer of porous materials, the porous medium of the filter membrane expands with a change in porosity. The change in porosity occurs due to a decrease in the pore volume of the porous environment, since the solid parts together with the liquid penetrate into the porous channels of the porous environment and hover in them. The conducted studies permitted the authors to identify and study the laws of the filtering process and establish the law of change in porosity of the porous environment. Based on the established law, a differential equation is derived. It allowed, for given initial and border-line conditions, stating the problem of filtering the liquid through a layer of solid particles of a variable porous medium of the filtering membrane. The solution of the non-stationary problem with initial and border-line conditions by the finite difference method allowed determining the hydrodynamic parameters of fluid filtration through a layer of particles of the porous environment of the filter membrane and to obtain a solution of the non-stationary boundary problem of fluid filtration in a deformed porous medium. The use of research results promotes complete clarification of the process fluid and thorough removal of the solid parts of valuable secondary raw materials of metal processing, in particular for powder metallurgy, facilitates the launching of waste-free production, and increases the level of environmental cleanliness in the operating area of cutting fluids.

Keywords: lubricoolants, solid parts, filtration, porous materials.

\section{Introduction}

In modern engineering industry during mechanical processing of machine parts, technological progress prompts the improvement of techniques with the aim of enhancing product quality and reducing its cost. At metalwork finishing operations, the use of cutting fluids is of particular importance. Machinebuilding enterprises use high-performance plant units and machine tools where the main technological element is liquid. During operation, process fluids are continuously and intensively contaminated with solid particles of metal processing.

\section{Formulation of the problem}

It is known that the solid parts of the slime, which was washed by the liquid into the contact zone of abrasive grains on the grinding wheel with the surface of the part, are deformed themselves and trigger the 
deformation of the material over the processed surface, which results in burns on the treated surfaces [1,2]. In order to exclude burns on the surfaces of parts during grinding, to reduce surface roughness and to maintain cutting fluids in an appropriate working condition, contaminated fluids must be purified from solid particles of metalworking. With bigger number and larger variety of cutting fluids used in mechanical engineering, the requirements for technology and equipment for process fluid cleaning systems are increasing.

\section{Discussion of modern reference publications}

To restore the original parameters and their properties, process fluids are purified of mechanical impurities. The most widely used methods of purification of process liquids are those done by filtration [3].

The use of filtering to purify and clarify process fluids is most effective, since with filtering through a layer of porous materials, it is possible to achieve complete extraction and removal of solids from liquids [4], [5], [6]. However, the structural features of the pores in the porous environment trigger a number of specific phenomena that arise when liquids move in the pore channels of the porous medium.

\section{The purpose and setting the goal of the study}

The purpose of the study is to research and establish the laws of filtering process fluids through porous materials. When filtering process liquids through a layer of porous materials, the porous medium of the filter partition changes with a change in its porosity. The change in porosity occurs due to a decrease in the pore volume of the porous space, since solid particles together with the liquid penetrate into the pores of the channels of the porous space and hover in it.

\section{Statement of the main research material}

In this model, the process of filtering slime suspension leads to gradual clogging of the pores of the filter. When filtering with gradual clogging of the pores the filter membrane with a volume of $W_{\phi}\left[\mathrm{m}^{3}\right]$, which contains a layer of granular porous material with porosity $\Pi$, is exposed to a continuous flow of technological fluid with a volume $W_{ж}\left[\mathrm{~m}^{3}\right]$ per minute, which contains solid parts with mass $\kappa_{3}[\mathrm{~kg}]$. The solid particles that enter the pores of the channels of the filter membrane hover and linger in it, with the filtered liquid continuing to move at the same speed. Solid particles that are stuck in the channel pores of the filter septum, change its porosity and affect the duration of the filtering process.

To determine the porosity of the filter membrane at any time and the phase of filtering the liquid through the porous layer of the filter membrane, it is necessary to know the mass of solid parts that are suspended in the pores of the loose porous material of the filter membrane. To do this, we take the time $t$ as an independent variable, and the unknown function $y(t)$ as the mass of solid parts that hang in the channel pores of the filter membrane in $t$ minutes after the start of filtering. Let us determine how much the mass of solid particles of impurities in the filter partition changes over a period of time from $t$ to the moment $(t+\Delta t)$.

In one minute $W_{\varkappa}\left[\mathrm{m}^{3}\right]$ of contaminated liquid enters the filter membrane, and in $\Delta t$ minutes $-W_{\varkappa} \cdot \Delta t$ $\left[\mathrm{m}^{3}\right]$, and in these $W_{\varkappa} \cdot \Delta t\left[\mathrm{~m}^{3}\right]$ of liquid there are $\kappa_{3} \cdot W_{\varkappa} \cdot \Delta t[\mathrm{~kg}]$ of solid particles of admixture. On the other hand, during the time $\Delta t$ as much as $W_{\mathscr{⿰}} \cdot \Delta t\left[\mathrm{~m}^{3}\right]$ of the filtered liquid flows through the filter membrane.

At time $t$, the entire filter membrane with a volume of $W_{\phi}\left[\mathrm{m}^{3}\right]$ contains $y(t)[\mathrm{kg}]$ of solid particles of impurities. Consequently, in the volume $W_{\phi} \cdot \Delta t\left[\mathrm{~m}^{3}\right]$, there could be $W_{\phi} \cdot \Delta t \cdot y(t)[\mathrm{kg}]$ of solid particles of impurities if during the time $\Delta t$ the amount of solid particles in the filter partition is not added. But since the mass of solid particles of impurities during this time increases by an amount $\delta$, infinitely small with $\Delta t \rightarrow 0$, then in the volume of liquid $W_{\varkappa} \Delta t\left[\mathrm{~m}^{3}\right]$ there will be $W_{\varkappa} \cdot \Delta t[y(t)+\delta][\mathrm{kg}]$ of solid particles of impurities, where $\delta \rightarrow 0$, at $\Delta t \rightarrow 0$.

Thus, the liquid entering the filtering membrane, over a period of time $(t, t+\Delta t)$ contains $\kappa_{3} \cdot W_{\varkappa} \cdot \Delta t$ $[\mathrm{kg}]$ of solid particles of impurities, and the volume of the porous layer of the filtering membrane taken by the liquid contains $W_{\varkappa} \cdot \Delta t[y(t)+\delta][\mathrm{kg}]$ of solid particles of impurities. 
The increment of solid particles of impurities over time $[y(t+\Delta t)-y(t)]$ is equal to the difference of these quantities, i.e.:

$$
[y(t+\Delta t)-y(t)]=\kappa_{3} W_{\varkappa} \cdot \Delta t-W_{\varkappa} \cdot \Delta t[y(t)+\delta] .
$$

We divide the resulting equation by $\Delta t$ and proceed to the limiting value with $\Delta t \rightarrow 0$. On the left side of the equation we obtain the derivative $y^{\prime}(t)$, and on the right $-\kappa_{3} W_{\varkappa}-W_{\varkappa} y(t)$, since $\delta \rightarrow 0$,.with $\Delta t \rightarrow 0$.

Therefore, we have a differential equation:

$$
y^{\prime}(t)=\kappa_{3} W_{\varkappa}-W_{\varkappa} y(t) .
$$

The differential equation is a linear homogeneous differential equation [7]. We solve these linear homogeneous differential equations by the method of divisible variables. To solve linear homogeneous differential equation (1) we bring it to the form:

$$
y(t)=c \cdot e^{-W_{\supset c} \cdot t}+\kappa_{3} .
$$

Since at the initial moment of filtration, with $t=0$, there were no solid parts of impurities in the filter membrane, their mass was $y(0)=0$, assuming in equation (2) $t=0$, we find the integration constant $c: c=-\kappa_{3}$.

Presenting the value $c$ into the equation (2), we obtain:

$$
y(t)=\kappa_{3}\left(1-e^{-W_{x c} \cdot t}\right) .
$$

With $t=t_{\kappa}$, in porous layer of the filtering membrane there are:

$$
m_{T}=\kappa_{3}\left(1-e^{-W_{x c} \cdot t}\right),
$$

where $m_{T}-$ the mass of solid particles, $[\mathrm{kg}]$.

The derived formula (3) makes it possible to determine the mass of solid parts that are suspended and hovering in the channel pores of loose porous material of the filter membrane at any time during the process of filtering a lubricoolant fluid with solid particles of metal working and subsequently derive the law of change in porosity of a porous medium based on the following reasoning.

Assuming that the change in porosity is proportional to the increase in the mass of solid parts in the porous layer of the filter membrane with volume $W_{\phi}, \mathrm{m}^{3}$, we obtain:

$$
d \Pi=\frac{d m_{T}}{\rho \cdot W_{\phi}},
$$

where $\rho-$ the density of porous medium, $\left[\mathrm{kg} / \mathrm{m}^{3}\right]$.

Since when filtering water-slurry suspensions through a filter membrane the porous medium continuously changes and increases due to solid particles of slurry, the continuity equation takes the form:

$$
\frac{\partial\left(\rho_{0} u\right)}{\partial x}+\frac{\partial\left(\rho_{0} v\right)}{\partial y}+\frac{\partial\left(\rho_{0} w\right)}{\partial z}+\Pi \frac{\partial \rho}{\partial t}+\rho \frac{\partial \Pi}{\partial t}=0
$$

Substituting the value in the continuity equation:

$$
\frac{\partial \rho}{\partial t}=\frac{d \rho}{d P} \cdot \frac{\partial P}{\partial t} ; \quad \frac{\partial \Pi}{\partial t}=\frac{d \Pi}{d m_{T}} \cdot \frac{\partial m_{T}}{\partial t},
$$

we receive:

$$
\frac{\partial\left(\rho_{0} u\right)}{\partial x}+\frac{\partial\left(\rho_{0} v\right)}{\partial y}+\frac{\partial\left(\rho_{0} w\right)}{\partial z}+\Pi \frac{d \rho}{d P} \frac{\partial P}{\partial t}+\rho \frac{d \Pi}{d m_{T}} \frac{\partial m_{T}}{\partial t}=0 .
$$

The real liquid is poorly compressible, therefore, with a sufficient degree of accuracy we can present as follows:

$$
\rho-\rho_{o}=\frac{\rho}{\alpha}\left(P-P_{o}\right)
$$

where $\alpha$-modulus of elasticity, $\left[\mathrm{N} / \mathrm{m}^{2}\right]$. 
Determining via the formulas (3), (4) and (7) the numerical values of:

$$
\begin{gathered}
\frac{\partial m_{T}}{\partial t}=W_{\varkappa c} \cdot \kappa_{3} \cdot e^{-W_{\jmath c} \cdot t} ; \\
\frac{d \Pi}{d m_{T}}=\frac{1}{\rho \cdot W_{\phi}} ; \\
\frac{d \rho}{d P}=\frac{\rho}{\alpha},
\end{gathered}
$$

after appropriate substitutions, the continuity equation for a variable porous medium takes the following form:

$$
\frac{\partial\left(\rho_{0} u\right)}{\partial x}+\frac{\partial\left(\rho_{0} v\right)}{\partial y}+\frac{\partial\left(\rho_{0} w\right)}{\partial z}+\Pi \frac{\rho}{\alpha} \frac{\partial P}{\partial t}+\frac{\kappa_{3} \cdot W_{\varkappa c} \cdot e^{-W_{\jmath c} \cdot t}}{\rho \cdot W_{\phi}}=0 .
$$

To solve the obtained differential equation (11) when filtering a slurry-water suspension through a porous layer of a filter membrane, we assume that the fluid moves in one direction according to the accepted model of the slurry-water filtering process. Then equation (11) takes the form:

$$
\frac{\partial\left(\rho_{0} w\right)}{\partial z}+\Pi \frac{\rho}{\alpha} \frac{\partial P}{\partial t}+\frac{\kappa_{3} \cdot W_{\mathcal{~}} \cdot e^{-W_{\mathcal{~} c} \cdot t}}{\rho \cdot W_{\phi}}=0
$$

The process of filtering water-slurry suspensions through a porous layer of solid parts of the filter membrane obeys the linear law of filtration - Darcy's law [8], [9]:

$$
w=k_{\phi} \cdot \frac{d P}{d z}
$$

where $k_{\phi}$ - coefficient of filtration, [m/s].

The filtration coefficient has a dimensionality of speed and characterizes the properties of the filter material for a particular type of liquid. It should be noted that it is desirable to determine the value of filtering coefficient in experimental way, since the proposed theoretical formulas do not always give sufficiently accurate results [10].

Performing the corresponding transformations of the filtering equation for a variable of porous medium through pressure fluctuations on the filter membrane, we obtain the final form of this equation for filtering liquid in a variable porous medium:

$$
\frac{\partial^{2} P}{\partial z^{2}}+\Pi \frac{\rho \cdot g}{\alpha \cdot k_{\phi}} \frac{\partial P}{\partial t}+\frac{\kappa_{3} \cdot g \cdot W_{\varkappa} \cdot e^{-W_{\jmath c} \cdot t}}{k_{\phi} \cdot \rho \cdot W_{\phi}}=0 .
$$

Thus, basing on the law of changing the porosity of the filter membrane, the differential equation for filtering the liquid in a variable porous medium is derived. Differential equation (14) allows, for given initial and limiting conditions, to obtain a solution to the problem of filtering fluid through a layer of solid parts of a variable porous medium of the filter wall in the time $0 \leq t \leq T$ and spatial $0 \leq z \leq L$ values:

$$
P(z, 0)=P_{1} ; \quad P(0, t)=P_{1} ; \quad P(L, t)=P_{2} .
$$

On the surface of the filter membrane, the pressure distribution is defined as a function of coordinates and time $P(z, t)$.

Pressure distribution on the surface of the filter membrane at the initial time of filtration:

$$
\text { with } t=0 \quad P(z, 0)=P_{l} .
$$

Since the pressure on the surface of the filter membrane is kept constant during filtration, the hydrostatic pressure force, which acts perpendicular to the surface of the filter baffle, is a constant value.

We introduce into the differential equation (14) a force that acts perpendicularly to the filter membrane. As a result, we obtain the differential equation of deformation in the porous medium of the filtering membrane:

$$
\frac{\partial^{2} F_{n}}{\partial z^{2}}+\Pi \frac{\rho \cdot g}{\alpha \cdot k_{\phi}} \frac{\partial F_{n}}{\partial t}+\frac{\kappa_{3} \cdot g \cdot S \cdot W_{\jmath} \cdot e^{-W_{\jmath c} \cdot t}}{k_{\phi} \cdot \rho \cdot W_{\phi}}=0
$$


Given the initial and boundary conditions of the filtering process of water-slurry suspensions, we arrive at the formulation of non-stationary boundary-value problem of filtering liquids in a deformed porous medium.

The solution of the non-stationary problem (14) with initial and boundary conditions (15) is carried out by the finite difference method [11], [12]. The essence of the method is that it is solved by the desired set of numbers at the corresponding points of a certain set, where they use the set of points of intersection of straight lines as a discrete set of points.

As a discrete set of numbers $D_{h}$, we use the set of intersection points of straight lines $z=m \cdot h$, $t=n \cdot \tau$, with $m=0, \pm 1, \pm 2$, and $n=0,1,2, \ldots,[T / \tau]$, where $h>0, \tau>0$ are some numbers.

We assume that step $\tau$ is related to step $h$ by the dependence $\tau=r h^{2}$, where $r=$ const, so the grid $D_{h}$ depends only on the parameter $h$. The desired grid function is the table $[P]_{h}=\{P(m h, n \tau)\}$ of the values of the solution $P(z, t)$ of equation (14) at the grid points $D_{h}$.

The implicit difference scheme approximating differential equation (17) has the form:

$$
\begin{gathered}
\frac{P_{m}^{n+1}-P_{m}^{n}}{\tau}+\Pi \frac{\rho \cdot g}{\alpha \cdot k_{\phi}} \cdot \frac{P_{m+1}^{n+1}-2 P_{m}^{n+1}+P_{m-1}^{n+1}}{h^{2}}+\frac{\kappa_{3} \cdot g \cdot W_{\text {ж }} \cdot e^{-W_{\text {эc }}}}{k_{\phi} \cdot \rho \cdot W_{\phi}}=0 ; \\
P_{m}^{0}=P_{1} ; \quad P_{0}^{n}=P_{1} ; \quad P_{L}^{n}=P_{2} .
\end{gathered}
$$

In order to calculate the value $P_{m}^{n+1}$ with $m=0,1,2, \ldots, L$, when the value $P_{m}^{n}$ is known with $m=0,1,2, \ldots, L$, it is necessary to solve the problem:

$$
\begin{gathered}
\frac{P_{m}^{n+1}}{\tau}-\frac{P_{m}^{n}}{\tau}+\Pi \frac{\rho \cdot g}{\alpha \cdot k_{\phi}} \cdot \frac{P_{m+1}^{n+1}-2 P_{m}^{n+1}+P_{m-1}^{n+1}}{h^{2}}+\frac{\kappa_{3} \cdot g \cdot W_{\text {жc }} \cdot e^{-W_{\text {эc }} \cdot t}}{k_{\phi} \cdot \rho \cdot W_{\phi}}=0 ; \\
P_{0}^{n+1}=P_{1} ; \quad P_{L}^{n+1}=P_{2} .
\end{gathered}
$$

After multiplying both parts of the difference equation by the factor $«-\tau »$ the problem will take the form:

$$
P_{m}^{n}-P_{m}^{n+1}-\tau \cdot \Pi \frac{\rho \cdot g}{\alpha \cdot k_{\phi}} \cdot \frac{P_{m+1}^{n+1}-2 P_{m}^{n+1}+P_{m-1}^{n+1}}{h^{2}}=\tau \cdot \frac{\kappa_{3} \cdot g \cdot W_{\mathcal{~}} \cdot e^{-W_{\text {эc }} \cdot t}}{k_{\phi} \cdot \rho \cdot W_{\phi}}=0 .
$$

Let us introduce the following denominations:

$$
\begin{gathered}
V_{m}=P_{m}^{n+1}, \quad a_{m}=\Pi \frac{\rho \cdot g}{\alpha \cdot k_{\phi}} r, \quad b_{m}=2 \Pi \frac{\rho \cdot g}{\alpha \cdot k_{\phi}} r-1, \quad c_{m}=\Pi \frac{\rho \cdot g}{\alpha \cdot k_{\phi}} r, \\
q_{m}=P_{m}^{n}-\frac{k_{3} \cdot g \cdot W_{\jmath c} \cdot e^{-W_{x c} \cdot t}}{k_{\phi} \cdot \rho \cdot W_{\phi}} \tau, \quad a_{0}=P_{1}, \quad \beta_{0}=P_{2} .
\end{gathered}
$$

After appropriate substitutions and transformations, taking into account the introduced denominations, the difference equation (19) with the boundary conditions (20) takes the form:

$$
\begin{gathered}
a_{m} \cdot V_{m-1}+b_{m} \cdot V_{m}+c_{m} \cdot V_{v+1}=q_{m}, \\
V_{0}=\alpha_{0} ; \quad V_{L}=\beta_{0},
\end{gathered}
$$

with $m=1,2,3, \ldots, L-1$.

The coefficients $a_{m}, b_{m}, c_{m}$ satisfy the conditions $a_{m}>0, c_{m}>0,\left|b_{m}\right|>a_{m}+c_{m}+\delta$, where $(\delta>0)$. Therefore, the problem has a unique solution.

To solve the difference boundary value problem, we write the equation $V_{0}=a_{0}$ of system (21), (22) in the form:

$$
V_{0}=M_{1 / 2} \cdot V_{1}+K_{1 / 2}
$$

where $M_{1 / 2}=0, K_{1 / 2}=a_{0}$.

From the equation $a_{1} \cdot V_{0}+b_{1} \cdot V_{1}+c_{1} \cdot V_{2}=q_{1}$, corresponding to number $m=1$ in the system $(21,22)$, we exclude $V_{0}$ using equation $V_{0}=M_{1 / 2} \cdot V_{1}+K_{1 / 2}$. 


\section{Vitalii Molchanov}

The result is written in the following form permitted in relation to $V_{0}$ :

$$
V_{1}=M_{3 / 2} \cdot V_{2}+K_{3 / 2}
$$

where $M_{3 / 2}=\frac{-c_{1}}{b_{1}}, K_{3 / 2}=\frac{a_{1} \cdot \alpha_{0}-q_{1}}{-b_{1}}$.

We continue the described exclusion process for values $m=2,3,4, \ldots$. The coefficients obtained in the process of eliminating the ratios $V_{m}=M_{m+1 / 2} \cdot V_{m+1}+K_{m+1 / 2}$, are calculated using recurrence formulas:

$$
M_{m+1 / 2}=\frac{-c_{m}}{b_{m}+a_{m} M_{m-1 / 2}} ; \quad K_{m+1 / 2}=\frac{q_{m}-a_{m} K_{m-1 / 2}}{b_{m}+a_{m} M_{m-1 / 2}} .
$$

The last of the formulas received in this way is as follows:

$$
V_{L-1}=M_{L-1 / 2} \cdot V_{L}+K_{L-1 / 2} \text {. }
$$

Since the value $V_{L}=\beta_{0}$, it is possible to calculate $V_{L-1}=M_{L-1 / 2} \cdot \beta_{0}+K_{L-1 / 2}$.

After that $V_{L-2}=M_{L-3 / 2} \cdot V_{L-1}+K_{L-3 / 2}$ and so forth, till $V_{1}$ is defined.

The obtained solution permits one to determine the hydrodynamic parameters of fluid filtration through a layer of particles of the porous medium in the filter membrane under given initial and boundary conditions and to obtain a solution of the non-stationary boundary problem of fluid filtration in a deformed porous medium.

\section{Conclusions}

1. The studies carried out permitted to identify and study the laws behind the process of filtering fluids through bulk porous materials.

2. The revealed patterns made it possible to establish the law of change in the porosity of the filter partition.

3. Based on the law of changing the porosity of the filter membrane, a differential equation is derived that allows, for given initial and boundary conditions, deriving a solution to the non-stationary boundary problem of filtering the liquid through the layer of solid parts of the variable porous environment of the filter membrane.

4. The introduction into the differential equation (14) of a force that acts perpendicular to the filter baffle permits to obtain a differential equation for the deformation of a porous medium.

\section{References}

[1] V. F. Molchanov, "Teoreticheskie issledovaniya veroyatnosti zaklinivaniya tverdyh chastic pri shlifovanii" ["Theoretical studies of the probability of jamming of solid particles during grinding"], Vestnik Zhitomirskogo gosudarstvennogo tehnologicheskogo universiteta. Seriya: Tehnicheskie nauki [Bulletin of Zhytomyr State Technological University. Series: Technical Sciences], no. 2 (80), pp. 114-119, 2017. [in Russian].

[2] L. V. Hudobin, and E. P. Gulnov, "Vliyanie zagryazneniya SOZh othodami shlifovaniya na prizhogoobrazovanie" ["Effect of lubricoolant contamination by grinding waste on burn formation"], Vestnik mashinostroeniya [Bulletin of Mechanical Engineering], no. 1, pp. 67-68, 1978. [in Russian].

[3] Yu. V. Polyanskov, and E. A. Karev, "K metodike issledovaniya effektivnoj ochistki SOZh ot mehanicheskih primesej pri abrazivnoj obrabotke" ["On the methodology for the study of effective cleaning of lubricoolant of mechanical admixture during abrasive treatment"], Trudy Ulyanovskogo politehnicheskogo instituta [Proceedings of the Ulyanovsk Polytechnic Institute], vol. 1, pp. 46-54, 1976. [in Russian].

[4] V. F. Molchanov, "Issledovanie filtracii zhidkostej cherez poristye materially" ["The study of fluid filtration through porous materials"], in Proc. of Scientific Conference "Matematicheskie problemy tehnicheskoj mehaniki" ["Mathematical problems of technical mechanics"], Dneprodzerzhinsk, Ukraine, 2003, pp. 71-72. [in Russian].

[5] V. F. Molchanov, "Postanovka i reshenie nestacionarnoj zadachi filtracii zhidkostej v poristoj srede" ["Formulation and solution of the non-stationary problem of fluid filtration in a porous medium"], Vestnik Harkovskogo nacionalnogo avtomobilno-dorozhnogo universiteta $i$ Severo-Vostochnogo nauchnogo centra Transportnoj akademii Ukrainy [Bulletin of the Kharkov National Automobile and Highway University and the Northeast Scientific Center of the Transport Academy of Ukraine], vol. 33, pp. 112-115, 2006. [in Russian]. 
[6] V. F. Molchanov, "Postanovka nestacionarnoj zadachi filtracii zhidkostej v poristoj srede" ["The formulation of the non-stationary problem of fluid filtration in a porous medium"], Matematicheskoe modelirovanie [Mathematical modeling], no. 1, pp. 28-30, 2014. [in Russian].

[7] L. S. Pontryagin, Obyknovennye differencialnye uravneniya [Ordinary Differential Equations]. Moscow, Russia: Nauka Publ., 1977. [in Russian].

[8] A. Shejdegger, Fizika techeniya zhidkostej cherez poristye sredy [Physics of the flow of fluids through porous media]. Moscow, Russia: Gostoptekhizdat Publ., 1960. [in Russian].

[9] V. F. Molchanov, "Issledovanie propusknoj sposobnosti filtrovalnyh setok" ["Investigation of filter grids throughput"], Sbornik nauchnyh trudov Dneprodzerzhinskogo gosudarstvennogo tehnicheskogo universiteta (tehnicheskie nauki) [Collection of scientific papers of the Dneprodzerzhinsk State Technical University (technical sciences)], vol. 3 (20), pp. 61-65, 2012. [in Russian].

[10] V. A. Zhuzhikov, Filtrovanie. Teoriya i praktika razdeleniya suspenzij [Filtration. Theory and practice of separation of suspensions]. Moscow, Russia: Himiya Publ., 1968. [in Russian].

[11] S. K. Godunov, and V. S. Ryabenkij, Raznostnye shemy. Vvedenie v teoriyu [Difference schemes. Introduction to Theory]. Moscow, Russia: Nauka Publ., 1977. [in Russian].

[12] V. F. Molchanov, "Postanovka i reshenie nestacionarnoj granichnoj zadachi filtracii zhidkostej v poristoj srede" ["Statement and solution of the non-stationary boundary value problem of fluid filtration in a porous medium"], Sbornik nauchnyh trudov Dneprovskogo gosudarstvennogo tehnicheskogo universiteta (tehnicheskie nauki) [Collection of scientific papers of the Dniprovsky State Technical University (technical sciences)], vol. 1 (32), pp. 53-58, 2018. [in Russian]. 